\title{
Der Physiologus und die Tierkunde der Griechen
}

\begin{abstract}
Introduction: Physiologus and its Relation to Ancient Zoology. 2. Brief introduction to Physiologus (chapter 5). 3. Aristotle's Zoology: a) Overview, b) Relation to philosophy, c) Presentation of De partibus animalium 1,5: Zoological research as an ideal image of bios theoretikós, contact with the religious. Conjecture: the of-

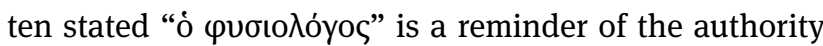
of Aristotle. 4. Hellenistic animal literature: a) the creation of reference works, b) the penetration of entertainment and mirabilia literature, c) the "occultism" of Bolos of Mendes. 5. Hellenistic philosophy: a) intelligence and character, legal relationship, b) the controversy Stoicism Academy (Philon, Plutarch). 6. Religious animal symbolism: a) Egyptians: Justification of the Egyptian animal cult by Hellenistic Egyptians of the Ptolemaic period. Presentation of Plutarch, De Iside et Osiride 74-76 (the symbolism mentioned here references the Greek mentality). Plutarch treats the subtleties of the symbolism with slight mockery, but on the philosophical level he accords animals very a prominent position as symbolic figures, because they are carriers of life and thus mirrors of the divine. b) Jews: Hellenized Jews try to justify the Old Testament prohibitions on certain foods ("impure animals") by symbolic interpretations. Letter of Aristeas 142-169; Philo, De specialibus legibus 4,100-118. c) Christians: Letter of Barnabas 10. Jewish dietary laws are not merely explained by symbolic interpretation, but also abolished.
\end{abstract}

\section{Einleitung: Der Physiologus und sein Bezug zur antiken Tierkunde}

Der Verfasser des Physiologus hat seine Aussagen über Eigenschaften der Tiere offensichtlich zum großen Teil aus der ausgedehnten Tierliteratur der griechisch-römischen Antike geschöpft. Diesem Hintergrund in der literarischen Tradition soll dieser Beitrag nachgehen. Die Forschung hat schon zu vielen Einzelheiten im Physiologus literarische Parallelen nachgewiesen. Eine klassische Quellenhypothese hat Max Wellmann (1930) entwickelt: Der Autor habe seine naturkundlichen Aussagen aus der (erschlossenen) Schrift eines Didymos von Alexandrien entnommen und dieser habe im wesentlichen die magisch-symbolische Naturkunde des Bolos von Mendes (um 200 v. Chr.) wiedergegeben. Die christlichen Deutungen müßte der Autor selbst hinzugefügt haben. Er weise auf seine Quelle immer wieder mit formelhaften Sätzen hin, am Anfang: „Der Phy- siológos hat gesagt“, am Ende: „Gut hat der Physiológos gesprochen. “1

Die zuletzt genannte Feststellung ist sicher richtig.

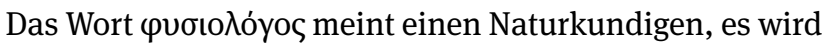
oft auf die vorsokratischen Philosophen angewendet. Es dürfte darin das Sinnelement einer alten, geachteten Autorität liegen, auf die man sich berufen kann. Es ist bezeichnend, daß es nicht kollektiv heißt: „die Naturkundigen sagen“ - dann würde man an gegenwärtige Fachleute denken -, sondern in einem emphatischen Singular „der Naturkundige“. Daß das Wort zum Titel der Schrift geworden ist, ist natürlich mißverständlich und sicher nicht im Sinne des Autors.

Nun sind gegen Wellmanns Hypothese aber viele Einwände erhoben worden und sie ist trotz des aufgewendeten Scharfsinns heute nicht mehr zu halten. ${ }^{2}$ Ein allgemeines Argument ist, daß manche Tiergeschichten offenbar aus der christlichen Deutung entwickelt sind, ${ }^{3}$ z. B. die Geschichte vom Einhorn (Kap. 22), das sich nur zähmen lasse, wenn es in den Schoß einer reinen Jungfrau springe. Dafür gibt es keine außerchristliche Parallelüberlieferung; es ist eine Erfindung, die die Jungfrauengeburt Christi verbildli-

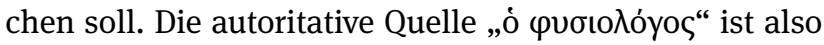
wohl eine Fiktion; man muß damit rechnen, daß der Autor den Stoff aus verschiedenen Quellen gesammelt, wenn nicht durch Verbindung griechischer Naturkunde mit biblischer Tradition selbst geschaffen hat.

Die Verfolgung einzelner Erzählmotive und ihrer Parallelen ergibt nur selten ein deutliches Bild von ihrer Herkunft. Darum scheint es fruchtbarer, die verschiedenen Bereiche der Tierliteratur in ihrer Eigenart und Entwicklung durchzugehen und von hier aus zu fragen, welche Spuren sie im Physiologus hinterlassen haben. Man kann im Großen vier Bereiche ansetzen:

- die Zoologie des Aristoteles,

- die Tierbücher des Hellenismus,

- die Mensch-Tier-Diskussion in der Philosophie,

- die religiöse Tiersymbolik in ägyptischem, jüdischem und christlichem Kontext.

Der Physiologus knüpft an die letztere an, und daß diese in Ägypten beheimatet ist, paßt zu der öfters gemachten

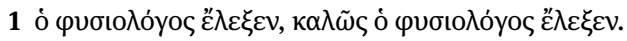

2 Ausführliche Diskussion bei Alpers (1984).

3 So schon Lauchert (1889, 4); dann Alpers (1984, 25-30, 35-41).
} 
Beobachtung, daß manche Einzelheiten in dieser Schrift auf eine Entstehung in Ägypten deuten.

\section{Kurze Vorstellung des Physiologus (Kapitel 5)}

$\mathrm{Zu}$ Beginn scheint es jedoch angebracht, an einem Beispiel deutlich $\mathrm{zu}$ machen, was die typischen Elemente des Physiologus sind, auf die sich die Beobachtungen beziehen sollten. Dafür sei Kap. 5 „Vom Nachtraben“ (Пєрі̀

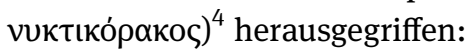

\begin{abstract}
Der Psalmist sagt: „Ich ward wie ein Nachtrabe an einer Ruinenstätte“ [Ps 102,7]. Der Physiologos sagte vom Nachtraben, daß dieser Vogel die Nacht mehr liebt als den Tag. So hat auch unser Herr Jesus Christus uns geliebt, die wir in Finsternis und Todesschatten saßen [Jes 9,2; Mt 4,16; Lk 1,79], uns, das Volk der Heiden - mehr als das Volk der Juden, welche die Sohnschaft und die Verheißung der Väter ererbt hatten. Darum sagte der Erlöser auch: „Fürchte dich nicht, du kleine Herde, denn es gefiel dem Vater, euch das Reich zu geben“ [Lk 12,32] und so fort. Indessen wirst du mir sagen, daß der Nachtrabe unrein ist gemäß dem Gesetz [Dtn 14,17]. Wieso wird ihm dann die Rolle des Erlösers gegeben? Gut sagt der Apostel: „Ihn, der keine Sünde kannte, hat er um unseretwillen zur Sünde gemacht“ [2 Kor 5,21]. Er hat sich selbst erniedrigt, um uns alle zu retten und damit wir erhöht würden. Gut hat also der Physiologos gesprochen über den Nachtraben. ${ }^{5}$
\end{abstract}

\footnotetext{
4 vขктıко́ $\alpha \xi$ wird in Ps 102,7 $\left(101,7^{\mathrm{LXX}}\right)$ und Dtn 14,16 von Luther mit „Käuzchen“ übersetzt; dem folgen neuere PhysiologusÜbersetzungen. Aber das Käuzchen heißt griechisch y $\lambda \alpha \tilde{u} \xi$ (oft auf Münzen von Athen abgebildet). Der „Nachtrabe“ wird mehrmals bei Aristoteles erwähnt; seine Angaben passen am besten auf die Waldohreule: Asio otus L., dazu Aubert und Wimmer $(1868,113)$; Thompson $\left.{ }^{2} 1936,207-209\right)$. Bei anderen Autoren kann mit dem „Nachtraben“ ein anderer Eulenvogel gemeint sein, so Strabo, Geographica 17.24: „,von der Größe eines Adlers“, wohl der Uhu.

5 Text nach Sbordone (1936a, 19-22): „Фпซìv ò $\Psi \alpha \lambda \mu \omega \delta$ ós.

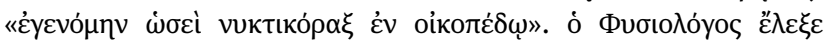

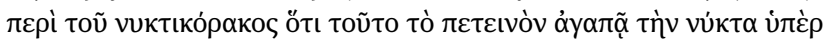

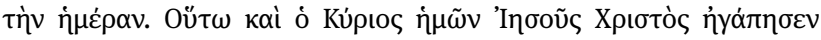

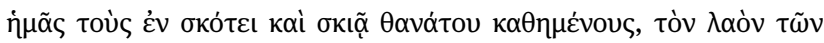

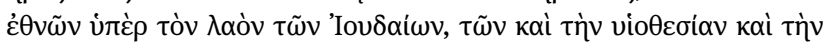

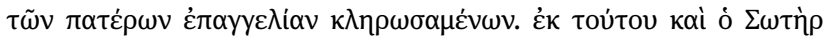

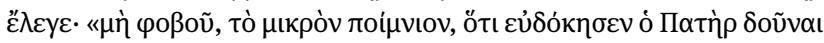

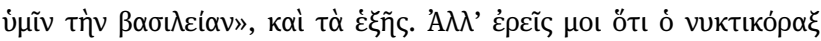

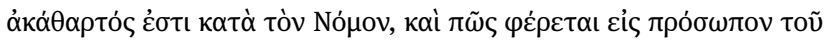

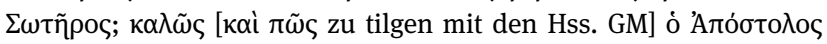

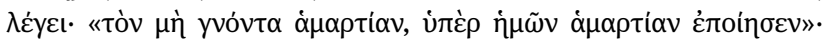

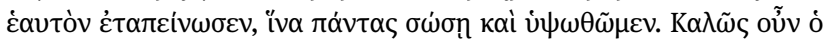

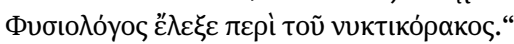

Der Autor geht von einer Psalmstelle aus, wo der Dichter sich mit einem „Nachtraben“ vergleicht, der an einer verödeten Stätte haust. Als zweites Element folgt eine naturkundliche Aussage des Physiológos: Dieses Tier liebe die Nacht mehr als den Tag. Eine entsprechende Aussage findet sich bei Aristoteles in der Historia animalium 9.34.619b18-23:

\begin{abstract}
Die Kauze und die Nachtraben und alle anderen Tiere, die bei Tag nicht zu sehen vermögen, beschaffen sich durch Jagd in der Nacht ihre Nahrung; allerdings tun sie das nicht die ganze Nacht lang, sondern bei Einbruch der Abenddämmerung und bei der Morgenröte. Sie jagen Mäuse, Eidechsen, Sphondylai [?] und andere Kleintiere dieser Art. ${ }^{6}$
\end{abstract}

Hier wird ein Unterschied in der Sichtweise erkennbar: bei Aristoteles eine sachliche Beschreibung des Verhaltens, das Jagen bei Nacht, verbunden mit einer kausalen Erklärung, dem schwachen Sehvermögen bei Tag; ${ }^{7}$ im Physiologus das subjektive „Lieben“, das mit den folgenden Schriftzitaten harmoniert.

Auf die zwei Vorgaben, die biblische und die naturkundliche, folgt die theologische Deutung, die beides in

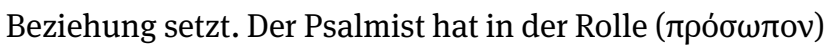
des Erlösers gesprochen (eine in der Psalmenexegese häufige Denkfigur), also liebt dieser die Nacht. Das scheint paradox; es ist so zu deuten, daß er die Menschen ,in Nacht und Todesschatten“, d.h. die Heiden, vor denen bevorzugt, die im Licht stehen, nämlich den Juden. Die Psalmstelle deutet also verschlüsselt auf die Ablösung der Juden als erwähltes Volk durch die Heiden. Dieser Vorgang wird mit einer weiteren Bibelstelle belegt, nach der ,das Reich (Gottes)“ vom Vater einer kleinen Herde gegeben wird. Diese exegetisch kühne Stellenkombination wird in großer Kürze, fast in einem Notizenstil, entworfen. ${ }^{8}$

Es folgt ein Einwand; man scheint fast einer theologischen Debatte beizuwohnen. Der Nachtrabe wird im Deuteronomium zu den unreinen Tieren gerechnet; wie kann

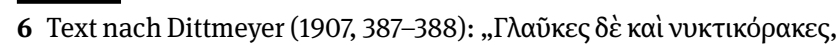

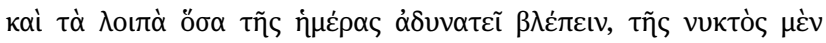

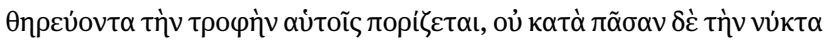

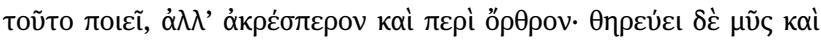

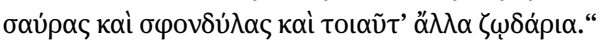

7 Nach neueren Erkenntnissen ist das Sehvermögen der Eulenvögel bei Tage durchaus nicht schwach; allerdings ist das Dämmerungssehen besonders hoch entwickelt. Brehm ('1879, 92); Grzimek (1970, 378). Eigentlich ist also die Formulierung des Physiologus treffender als die des Aristoteles.

8 So ist nicht leicht zu verstehen, warum mit der „kleinen Herde“ (Lk 12,32) die Heiden gemeint sein sollen. Diese sind zwar bei Lukas kurz vorher erwähnt $(12,30)$, aber als Gegenbeispiel, nicht als Empfänger der Verheißung. 
er dann Christus symbolisieren? Die Lösung wird mit zwei Paulus-Zitaten gegeben, die von der Dialektik der Menschwerdung sprechen: Der Erlöser hat sich erniedrigt, ist „,zur Sünde“ (also „unrein“) geworden, um uns von der Sünde $\mathrm{zu}$ retten. Auch das ist eine kühne Wendung, die eigentlich mit der ursprünglichen Deutung über Kreuz steht. Die unausgesprochene Pointe des Kapitels ist ja, daß ein unheimliches Nachttier ${ }^{9}$ zum Symbol des Erlösers wird; das wird zunächst damit erklärt, daß die Liebe zur Dunkelheit die Liebe zu den Heiden symbolisiert. Jetzt erhält die Dunkelheit einen anderen Sinn; sie ist „die Sünde“, zu welcher der Erlöser in paradoxer Weise geworden ist. Der Verfasser scheint zu experimentieren; er versucht mehrere Möglichkeiten, ein Paradoxon zu deuten; Naturkunde und Bibel bieten ihm dafür das Material.

\section{Zoologie des Aristoteles}

Von den ältesten tierkundlichen Schriften der Griechen, etwa denen Demokrits, ist wenig bekannt; sie wurden alle durch die überragenden Arbeiten des Aristoteles verdrängt. Aristoteles hat die Zoologie als wissenschaftliches Fach begründet; seine Schriften darüber machen mehr als ein Drittel des Umfangs seiner erhaltenen Werke aus. Er sammelte ein umfangreiches Faktenmaterial; dafür beutete er ältere Literatur aus und holte systematisch Informationen von Jägern, Tierzüchtern, Fischern und Tauchern ein. In den Jahren 348-342 hielt er sich in Assos (Kleinasien) und Mytilene (Lesbos) auf; dort scheint er intensive zoologische Beobachtungen gemacht zu haben, wie man aus Ortsangaben in seiner Historia animalium schliesst. ${ }^{10}$ Insgesamt werden in diesen Werken über 500 Tierarten behandelt. Er führte anatomische Studien mit Hilfe eigener Sektionen durch und fertigte einen anatomischen Atlas an. In der Auswertung dieses Materials entwickelte er Grundzüge einer Systematik, die in wesentlichen Zügen noch heute gilt; manche seiner Klassenbezeichnungen leben in lateinischer Form weiter, z. B. Ěvto $\mu \alpha$ - lateinisch „insecta“, deutsch „Kerbtiere“. Er untersuchte physiologische Funktionen z. T. in eigenen Monographien, so die Fortbewegung (De incessu animalium und De motu animalium) und die Fortpflanzung (De generatione animalium). Vergleichende Anatomie wird in De partibus animalium behandelt. Aristoteles legte großen Wert auf ein Verständ-

\footnotetext{
9 So heißt es z. B. in einem Epigramm des Nikarchos (Anthologia

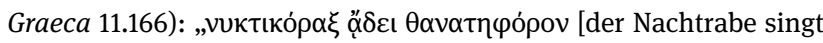
ein todbringendes Lied].“ 10 Leroi (2014).
}

nis von Kausalzusammenhängen. Gegen Legenden war er sehr kritisch; so bestritt er, daß das Wiesel seine Jungen aus dem Mund gebiert, ${ }^{11}$ daß die Hyäne jährlich ihr Geschlecht wechselt, ${ }^{12}$ daß der Elefant keine Gelenke in den Beinen hat und deshalb im Stehen, an einen Baum gelehnt, schlafen muß. ${ }^{13}$ Diese drei Legenden haben sich trotz seiner Autorität gehalten und tauchen mit Variationen auch im Physiologus auf (Kap. 21, 24 und 43). Im ganzen ist die Leistung des Aristoteles überwältigend; zwar sind ihm manche Fehler unterlaufen und viele seiner Versuche, allgemeine Regeln und Kausalzusammenhänge herzustellen, lassen sich nicht halten; aber dagegen steht eine Fülle von richtigen Beobachtungen und Einsichten.

Daß Aristoteles so viel Energie für die Zoologie aufgewendet hat, hängt mit seiner Vorstellung von Wissen und Wissenschaft zusammen. Platon hatte als eigentliches Objekt der Erkenntnis ein transzendentes Seiendes, die Ideen, erklärt; Aristoteles setzt an dessen Stelle die

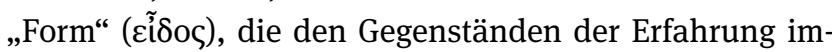
manent ist. Daraus folgt eine Hinwendung zur Empirie. Die Tierwelt erwies sich als besonders geeignetes Feld für die Anwendung seiner ontologischen Grundbegriffe:

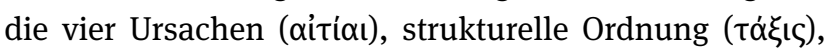

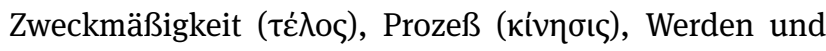

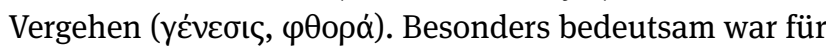
ihn, daß die artspezifische Struktur eines Lebewesens durch alle Prozesse erhalten bleibt und in der Fortpflanzung weitergegeben wird; hier ist die Dynamik des عĩos wirksam, und es wird sogar etwas Unsterbliches und Göttliches faßbar. ${ }^{14}$

Über das philosophische Interesse hinaus hat Aristoteles sich für zoologische Forschungsarbeit als solche begeistert. In einem berühmten und vielzitierten Kapitel ( $D e$ partibus animalium 1.5$)^{15}$ spricht er davon, daß man das Kleine, Geringfügige nicht verachten soll im Vergleich mit erhabenen Gegenständen wie der Sternenwelt. Der Astronom mag sich rühmen, mit göttlichen Sternwesen zu tun zu haben, aber die Gegenstände der Zoologie sind greifbarer, sie sind uns näher und unserer Natur verwandter

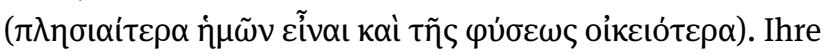
Objekte mögen für die sinnliche Wahrnehmung manch-

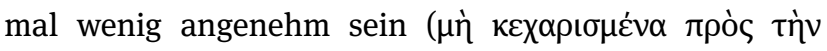
aı̈ $\sigma \theta \eta \sigma v)$ - da drängt sich der Gedanke an einen Aris-

\footnotetext{
11 De generatione animalium 3.6.756b13-757a2. 12 Historia animalium 6.32.579b16-30; De generatione animalium 3.6.757a2-13. Aus dem Wortlaut geht hervor, daß Aristoteles selbst die Genitalien einer Hyäne untersucht hat.

13 Historia animalium 2.1.498a9; De incessu animalium 9.709a9-10. 14 De anima 2.4.415a26-b7; De generatione animalium 2.1.731b24-35. 15 Den griechisch-deutschen Text s. Balss (1943, 8-13).
} 
toteles auf, der die Genitalien einer toten Hyäne inspiziert, umgeben von deren berüchtigtem Gestank -, aber ihre Betrachtung $(\theta \varepsilon \omega \rho i \alpha)$ bringt dem Forscher unermeßliche

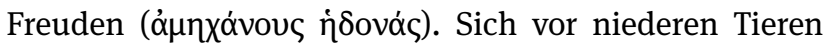
zu ekeln ist kindisch. Von Heraklit wird die Anekdote erzählt, er sei von Besuchern im Winter angetroffen worden, als er sich in dem schmutzigen Heizraum einer Bäckerei wärmte; da habe er gesagt: „Kommt nur herein, auch hier sind Götter!“ In jedem Tier steckt etwas Natürliches und Schönes, denn hier herrscht nicht blinder Zufall, sondern

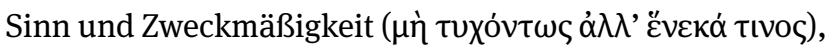
und das ist es, was Schönheit ausmacht. Auch der Mensch besteht aus abstoßenden Dingen, Blut, Knochen, Adern; seine Schönheit liegt in der Ganzheit der Gestalt.

Dieses Kapitel wirkt wie ein Hymnus auf die Tätigkeit des Zoologen; es erinnert an die Darstellung des ßíos $\theta \varepsilon \omega \rho \eta \tau$ menschlichen Lebens dargestellt wird. Und ebenso wie dort läßt Aristoteles ein religiöses Motiv anklingen: In der Mitte steht die Heraklit-Anekdote mit dem Spruch „Auch hier sind Götter.“ Im Studium der Tiere begegnet man dem Göttlichen. Man darf das wohl als ein persönliches Bekenntnis lesen.

Schließlich zwei Bemerkungen zum Physiologus. Dessen Autor hat wohl kaum Aristoteles selbst gelesen; direkte Berührungen (wie beim Nachtraben) sind selten. Aber Exzerpte daraus kann er gekannt haben (davon später), und der Ruf des großen Forschers mag zu ihm gedrungen sein. Könnte es nicht sein, daß hinter der Bezeichnung

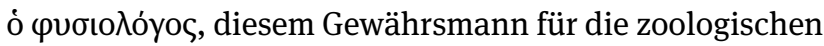
Tatsachen, die legendär gewordene Figur des Aristoteles steht? Dieser Gedanke ist gelegentlich geäußert, ${ }^{16}$ aber in neuerer Zeit nicht wieder aufgegriffen worden. Er würde aber den emphatischen Singular besser erklären als irgendeine obskure Quellenschrift. Ferner: Daß die Tierkunde für Aristoteles eine ins Religiöse gesteigerte Bedeutung hat, könnte eine Perspektive auf den Physiologus eröffnen. Freilich, eine echte Traditionslinie ist nicht zu verfolgen; in der griechischen Philosophie hat das keine Nachfolge gehabt. Allenfalls bei Plutarch könnte man ein Gegenstück sehen; aber da geht es um Tiersymbolik, nicht um Tierforschung. Immerhin ist der Gedanke gemeinsam, daß man im Blick auf die Tierwelt den Geheimnissen der Welt und des Göttlichen näherkommen könnte.

16 Ahrens (1885, 13); Lauchert (1889, 44); Goldstaub (1900, 346). Lauchert weist darauf hin, daß die von ihm benutzte Hand-

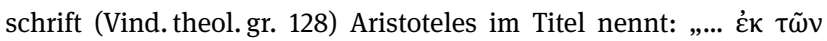

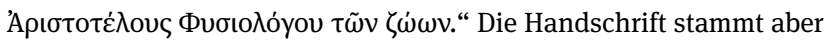
erst aus dem 14. Jh.

\section{Hellenistische Tierliteratur}

Die zoologischen Schriften des Aristoteles laden eigentlich zur Ergänzung, Nachprüfung, Korrektur ein. Aber in der hellenistischen Tierliteratur finden sich nur wenige Autoren, die in diesem Sinne weiterarbeiten; Theophrast und Straton von Lampsakos wären dafür zu nennen. Stattdessen bemühte man sich, sein Material leichter benutzbar zu machen; es entstanden Exzerpte in Katalogform. Eines wird mit dem Titel Zwïkó zitiert; es lief anscheinend unter dem Namen des Aristoteles selbst um. Ein anderes, Пврі $\zeta \omega \omega \nu$, wurde von Aristophanes von Byzanz verfaßt; davon ist ein Stück erhalten. ${ }^{17}$ Dieser Aristophanes, der zweite Leiter der Bibliothek von Alexandria, war eigentlich literarisch interessiert; er wollte offenbar das aristotelische Material als Bildungswissen in handlicher Form zugänglich machen. Natürlich kam auch neues Wissen dazu; später spielte im römischen Bereich der Brauch der Tierhetzen im Zirkus eine gewisse Rolle: Dafür wurden in großem Stil wilde Tiere vor allem in Afrika gefangen, und man lernte ihr Verhalten genauer kennen. Solches Material ist in die erhaltenen Tierbücher der Kaiserzeit eingegangen; die bedeutendsten sind die des Plinius (Naturalis historia,

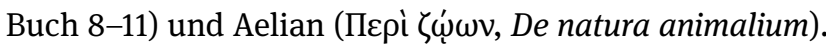
Solche Bücher hat der Verfasser des Physiologus sicher gekannt. Von ihnen kann er ein formales Element übernommen haben: die Katalogform. Geschlossene Kapitel werden gereiht, die oft stichwortartig mit dem Namen des Tieres anfangen. Bei Aristophanes gibt es besonders knappe, schnörkellose Aussagen fast nach Art eines Lexikons; die dem Physiológos zugeschriebenen Aussagen erinnern stilistisch daran.

Diese Tierbücher waren zunächst nur sachlich-informativ. Dann drang eine Tendenz ein, die man mit dem Begriff „Paradoxographie“ oder „Mirabilienliteratur“ bezeichnet. Da wurden Seltsamkeiten aller Art gesammelt; auch die Tierwelt hatte allerhand Bizarres und Anekdotisches zu bieten. Solche Tierbücher dienten weniger der Belehrung als der Unterhaltung. Verfasser solcher Bücher waren u.a. Antigonos von Karystos und Alexander von Myndos. Jetzt tauchten auch manche Legenden wieder auf, die Aristoteles bestritten hatte; davon war schon die Rede. Übrigens wurde ausgerechnet Aristophanes von Byzanz, der trockene Tier-Registrator, zum Opfer solcher erzählerischen Phantasie: Er soll sich in Alexandrien in eine Blumenverkäuferin verliebt haben, aber einen Riva-

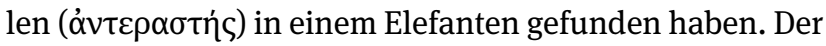
wurde öfters durch die Stadt geführt und jedesmal sei er

17 Herausgegeben von Lambros (1885). 
am Marktstand des Mädchens stehen geblieben, habe ihr mit dem Rüssel allerhand Leckereien gebracht und sei ihr dann unter das Hemd geschlüpft und habe ihre Brust gestreichelt. ${ }^{18}$ Diese Mirabilien-Literatur hat offensichtlich den Physiologus beeinflußt. Dessen Tiergeschichten haben oft ein Element des Erstaunlichen, Verblüffenden: z. B. die rührende Geschichte vom Pelikan, der die Jungen mit seinem Blut nährt (Kap. 4); von der Natter, deren Junges sich aus dem Bauch der Mutter herausfrißt und sie dadurch tötet (Kap. 10); vom Walfisch, der von Seefahrern für eine Insel gehalten wird, auf der sie landen können (Kap. 17).

Eine weitere Ausprägung der Tierkunde ist verbunden mit dem Namen des Bolos von Mendes (um 200 v. Chr.). Er interessierte sich für geheimnisvolle Wechselwirkungen, die er „Sympathie“ und „Antipathie“ nannte: Tiere, Pflanzen und Steine sind zu einem okkulten Wirkungszusammenhang verbunden; Krankheiten und ihre Behandlung spielen dabei eine große Rolle. Auch der Physiologus handelt manchmal von solchen Wirkungen; er flicht auch in die Reihe der Tiere einige Steine und Pflanzen ein (Diamant, Achat, Magnetstein, der sog. Gebärstein, der Peridexion-Baum, die Mandragora, die Sykomore). Die Lehre von okkulten Kräften klingt gelegentlich an. ${ }^{19}$

\section{Hellenistische Philosophie}

Das Verhältnis zwischen Mensch und Tier ist ein eigenes Thema. Seit archaischer Zeit wird in Tierfabeln menschliches Verhalten in Tieren gespiegelt; die attische Komödie spielt mit dem Gedanken (z.B. Aristophanes in den Vögeln), man denkt nach über die unterschiedliche natürliche Ausstattung von Mensch und Tier (etwa Protagoras in Platons gleichnamigem Dialog) und über eine mögliche Aufwärtsentwicklung des Menschen aus einem tierähn-

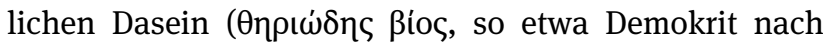
Diodor, Bibliotheca historica 1.8). Seit der Sophistenzeit gibt es verschiedene Meinungen über die Intelligenz der Tiere; man spricht von einem Wissen und Können, das sie nicht zu lernen brauchen, sondern das die Natur ihnen gibt (Xenophon, Kyropädie 2.3). Ein anderes Thema ist der Charakter: Manche Tiere sind tapfer, andere feige, sie können Dankbarkeit, Treue, Freundschaft zeigen. Bei Platon gibt es Reflexionen über eine naturhafte Vorstu-

18 Plutarch, De sollertia animalium 18; Plinius, Naturalis historia 8.13; Aelian, De natura animalium 1.38 .

19 Wellmann $(1930,19)$ führt fünf Stellen an; dies ist eine der Grundlagen seiner oben erwähnten Quellenhypothese. fe der Tugenden, die „tierhaft und sklavenhaft“ genannt werden kann (Politeia 430b8). Bei Aristoteles enthält die Historia animalium (Buch 8-9) eine große Materialsammlung zu Intelligenz und Charakter der Tiere; hier sind auch Studien des Theophrast in den Text eingeflossen.

Wenn man eine Wesensverwandtschaft zwischen Tier und Mensch annimmt, kann das praktische Folgen haben: Es wäre dann nicht erlaubt, sie zu töten, um sie zu essen. Der Vegetarismus war schon lange (wegen der Seelenwanderung) von Pythagoreern und Empedokles vertreten worden; jetzt wurde er auf einer ganz anderen Grundlage befürwortet. Wahrscheinlich geht auf Theophrast die Argumentation zurück, zwischen Mensch und Tier gebe es we-

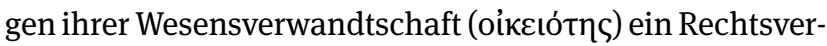
hältnis; die Tötung eines unschuldigen Tieres sei ein Unrecht, nur ein schädliches dürfe getötet werden. ${ }^{20}$

Die stoische Philosophie hat sich oft mit Tierpsychologie beschäftigt. In der Ethik wurde die Oikeiosis-Lehre mit der Berufung auf den Selbsterhaltungstrieb der Tiere und der Sorge für die Nachkommen illustriert. Jedoch

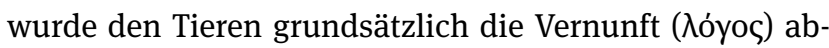
gesprochen; ihr intelligentes Verhalten sei nur eine QuasiVernunft. Chrysippos soll mehrmals die Symbiose der Steckmuschel ( $\pi i v v \alpha)$ mit einem Kleinkrebs, dem Muschel-

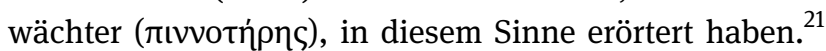
Poseidonios gab eine Analyse tierischen Verhaltens, die dem modernen Instinkt-Begriff sehr nahe kommt.

Dagegen entwickelte sich eine Opposition in der Akademie, vielleicht schon bei Karneades, wo auch den Tieren Logos zugesprochen wurde. Im 1. Jh. n. Chr. entwickelte sich zu diesem Thema eine Kontroverse, aus der zwei Texte erhalten sind: Philons von Alexandrien Dialog „Alexander oder über den Vernunftbesitz der Tiere“ (in armenischer Übersetzung erhalten) und Plutarchs Einleitung zu dem Dialog De sollertia animalium; aus späterer Zeit gehört dazu Porphyrios De abstinentia. Das philosophische Problem verband sich jetzt mit dem pythagoreischen Vegetarismus, und es wird sogar eine kosmische Dimension erkennbar: Die Stoiker dachten anthropozentrisch, die ganze Welt diene dem Interesse des Menschen; die Akademiker pluralistisch: Es gebe viele Wesen in der Welt, deren Interessen es verdienten, berücksichtigt zu werden.

Im Physiologus findet man wenige Spuren von solchen Mensch-Tier-Reflexionen. Natürlich ist von Intelligenzleistungen die Rede, der Fuchs kennt schlaue Tricks; es gibt moralisches Verhalten, die Elternliebe bei Pelikan und

20 Porphyrios, De abstinentia 2.22-24. Wie genau in dieser Paraphrase das Argument Theophrasts wiedergegeben ist, ist umstritten.

21 Hierüber wird in tierkundlichen Schriften oft gesprochen; ein Überblick etwa bei Gossen und Steier (1922, 1672-1673). 
Wiedehopf, die Monogamie bei der Turteltaube. Aber die Beziehung zum menschlichen Verhalten ist nur symbolisch, kein Zeichen von Verwandtschaft. Wenn der Biber, vom Jäger verfolgt, seine Geschlechtsteile opfert (Kap. 23), ist er ein Symbol für Keuschheit, aber nicht selber keusch.

Ein Gegenbeispiel findet man an ganz anderer Stelle in der frühchristlichen Literatur: Die Geschichte vom dankbaren Löwen. Sie ist in der Tierliteratur weit verbreitet: ${ }^{22}$ Ein entflohener Sklave namens Androklos trifft in der afrikanischen Wüste auf einen Löwen, der sich einen großen Dorn in den Fuß getreten hat und befreit ihn davon. Später wird dieser Androklos in Rom ad bestias verurteilt; in der Arena wird eben dieser Löwe auf ihn gehetzt; der erkennt ihn als seinen Wohltäter, wedelt mit dem Schwanz und leckt ihm Hände und Füße wie ein Hund. Diese rührende Geschichte ist in den apokryphen Paulus-Akten umgestaltet worden: Ein Löwe hatte sich in der Wüste von Paulus taufen lassen; später kommt es zu einer Begegnung in der Tierkampf-Arena und zu der dankbaren Wiedererkennung. Die Geschichte hat in christlichen Kreisen keinen großen Anklang gefunden; Hieronymus (De viris illustribus 7) lehnt den „getauften Löwen“ ausdrücklich ab, und der Text war verschollen, bis er aus einer koptischen Übersetzung und einem griechischen Papyrus rekonstruiert werden konnte. ${ }^{23}$

\section{Religiöse Tiersymbolik}

\section{1 Ägypter}

Religiöse Tiersymbolik, in Sprache und Bild, ist uralt und in vielen Kulturen verbreitet. Aber als bewußt angewendete Methode erscheint sie zuerst im Hellenismus im ägyptischen Bereich. Die Griechen waren früher vom ägyptischen Tierkult befremdet gewesen und hatten auch darüber gespottet; ${ }^{24}$ jetzt erscheinen explizite Erklärungen zum Symbolgehalt der verehrten Tiere, die zum Verständnis dieses Kultes beitragen können. Der ausführlichste erhaltene Text findet sich bei Plutarch, De Iside et Osiride $74-76,{ }^{25}$ der eine genauere Analyse verdient.

22 Gellius, Noctes Atticae 5.14 nach Apion; Plinius, Naturalis historia 8.56; Seneca, De beneficiis 2.19.1; Aelian, De natura animalium 7.48; Phaedrus, Fabulae 563 (Perry 1965, 526).

23 Schneemelcher ( $\left.{ }^{6} 1997,230,242\right)$; Stamatiou und Weckwerth (2010, 276-278).

24 Eine Liste von den Absurditäten der ägyptischen Tierverehrung gibt der Komiker Anaxandrides, frag. 40 (PCG 2).

25 Den griechisch-deutschen Text s. Görgemanns ( $\left.{ }^{2} 2009,258-267\right)$.
Eine Vorbereitung dazu gibt es schon in Kap. 10, das von der Hieroglyphen-Schrift handelt: Für „Himmel“ steht die Uräus-Schlange (áorí̧), weil sie nicht altert, ein Zeichen der Ewigkeit. Die Angehörigen des Kriegerstandes führen den Skarabäus (кóvӨ $\theta \rho \circ \varsigma)$ als Siegel, weil diese Käfer alle männlich sind. Das Verschlüsselte und Mysterienhafte dieser Bilder macht Plutarch dem griechischen Leser plausibel durch einen Vergleich mit den pythagoreischen $\sigma u ́ \mu \beta 0 \lambda \alpha$.

Nun zu Kap. 74-76. Der Tierkult hat zwei ernstzunehmende Gründe: Nützlichkeit und Symbolik. Für die Symbolik werden fünf Beispiele erläutert: Wiesel, Skarabäus, Uräus-Schlange, Krokodil, Ibis. Zu diesen Tieren einige Bemerkungen. Das Wiesel wird durch das Ohr begattet und gebiert aus dem Mund. (Plutarchs Bemerkung „das glauben und sagen viele noch immer“ zeigt, daß er von dem Widerspruch des Aristoteles wußte.) Das ist, wie Plutarch sagt, ein Gleichnis für „die Entstehung des Logos“ (

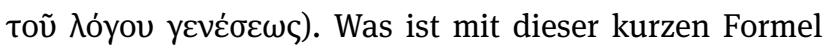
gemeint? Etwa, daß man mit dem Ohr eine Aussage aufnimmt und mit dem Mund wieder ausspricht? Das wäre die Weitergabe einer Information, aber nicht die „Entstehung“ des Logos. Vielmehr ist vielleicht in starker Verkürzung auf die stoische Theorie von der Entstehung der Erkenntnis angespielt. Sie geht von der Sinneswahrnehmung aus (dafür steht das Ohr), diese wird von der Ver-

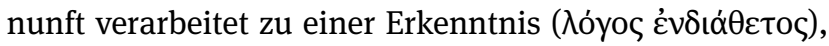
und diese wird sprachlich mit dem Mund geäußert ( $\lambda$ óyo $\pi \rho о \varphi о \rho ı к о ́ \varsigma)$. Die Symbolik würde also auf ein Lehrstück griechischer Philosophie hinauslaufen. Auch die Deutungen der anderen Tiere setzen griechisches Bildungsgut voraus. Die Bewegung des Skarabäus beim Rollen einer Kotkugel entspricht der doppelten Bewegung der Sonne: dem täglichen Umlauf von Ost nach West und dem jährlichen Umlauf von West nach Ost. Das ist ein elementares Lehrstück der griechischen Astronomie. Die UräusSchlange gilt als unsterblich; dieser Glaube findet sich auch sonst auf Schlangen bezogen (so im Physiologus 11); der Grund ist, daß die Häutung als Verjüngung verstanden wurde. Im Hellenismus ist die Schlange deshalb mit dem Zeitgott (Aion oder Chronos) verbunden; ihre göttlichen Eigenschaften werden ausführlich bei Philon von Byblos (FGH 790F4) dargestellt, der solche Deutungen den Phöniziern und Ägyptern zuschreibt. Das Motiv ist offenbar im hellenistischen Orient weit verbreitet. Das Krokodil, dieses gefährliche Raubtier, als göttlich zu erweisen, ist besonders schwierig; Plutarch führt vier Gründe an, darunter einen zahlensymbolischen: es legt 60 Eier, die Brutzeit ist 60 Tage, es lebt 60 Jahre. Die Zahl 60 als Grundlage des Sexagesimalsystems spielt in griechischen astronomi- 
schen Berechnungen eine wesentliche Rolle; das geht auf babylonische, nicht auf ägyptische Ursprünge zurück.

Diese Symbolismen sind also auf eine griechische, nicht auf eine ägyptische Vorstellungswelt abgestimmt. Plutarch führt sie aber ausdrücklich auf Ägypter zurück: „Man sollte sich nicht wundern, daß die Ägypter eine solche Liebe $\mathrm{zu}$ weit hergeholten Ähnlichkeiten [y $\lambda \dot{i} \sigma \chi \rho \alpha \iota$ ó zeichnung für symbolische Beziehungen, und y $\lambda$ ioxpos (mit der Grundbedeutung ,klebrig“) wird oft gebraucht, um mit leichtem Spott hartnäckige, gewaltsame, kleinliche Argumente und Fragen zu bezeichnen. ${ }^{26}$ Plutarch hat diese Deutungen also aus einer ägyptischen Quelle entnommen, der er kritisch, aber eher freundlich herablassend als ablehnend gegenübersteht. Wo diese Quelle zu suchen ist, läßt sich erraten: Deutungen der ägyptischen Religion in einem griechischen Denkhorizont gab es, seitdem dort mit den Ptolemäern die griechische Kultur dominierend geworden war; man war bestrebt, die einheimische Kultur nicht zu unterdrücken, sondern mit der griechischen auszugleichen. Ein frühes Beispiel ist Heka-

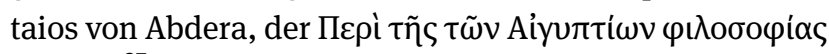
schrieb, ${ }^{27}$ auch gebürtige Ägypter wie Manethon von Sebennytos waren beteiligt. Dort war von Tieren wie Skarabäus, Schlange und Falke die Rede, in denen die Ägypter

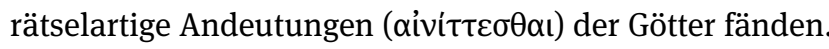
Auf solche Ägypter muß Plutarch sich beziehen; Max Wellmann hat vermutet, daß er eine Schrift des Ägypters Apion benutzt hat, die in der 1. Hälfte des 1.Jh. n. Chr. entstanden war. $^{28}$

Anknüpfend an diese „weit hergeholten Ähnlichkeiten“ entwickelt Plutarch dann ein tiefer greifendes Deutungsmuster. Symbolik, so sagt er, findet sich auch bei den Hellenen in Attributen der Götter, darunter auch Tiere; sogar Zahlen und geometrische Figuren haben (für die Pythagoreer) symbolisch-religiöse Bedeutung. Aber höher als die bildlichen Darstellungen bei den Griechen stehen lebendige Tiere als Symbolträger, weil sie beseelte Wesen sind. Schon in Kap. 74 (380F) hieß es, sie seien „undeutliche Bilder von der Wirksamkeit der Götter, so wie man in Regentropfen Bilder der Sonne sieht“; das wird jetzt $(76,382 \mathrm{~A})$ aufgegriffen: Sie sind „besonders klare,

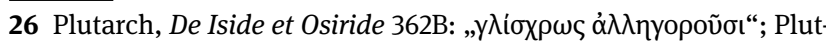
arch selbst hat eine bessere Erklärung. De recta ratione audiendi 43A:

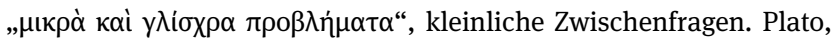
Politeia 488c, selbstironisch von einem gerade gebrauchten überraschenden Vergleich; Kratylos 414c, von verwegenen Etymologien, 435c von der Verlockung der Ähnlichkeit.

27 Diogenes Laertios Vitae philosophorum 1.10 (FGH 264F1).

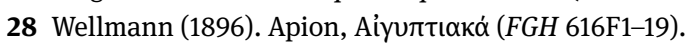

von der Natur geschaffene Spiegel“ des Göttlichen. Alles Beseelte, das die Fähigkeit der Selbstbewegung und des Erkennens besitzt, „hat einen Ausfluß und Anteil von der Schönheit des Denkenden in sich aufgenommen, ,von dem das Weltall gelenkt wird', wie Heraklit sagt." Hinter solchen Formulierungen steht die platonische Denkfigur von Vorbild und Abbild, Idee und Einzelding. Wesentliche

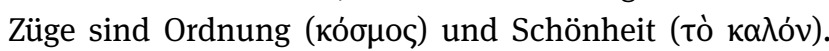
Man verehrt dann nicht die Tiere selbst (der ägyptische Tierkult als solcher ist also eine Verirrung), sondern durch sie das Göttliche. In diesen Stichworten klingt von ferne die Begeisterung des Aristoteles für die Zoologie an; auch Plutarch schwingt sich zu einem Hymnus auf Rang und Würde der lebendigen Tierwelt auf.

Gibt es von hier eine Verbindung zum Physiologus? Dieser hat keinen philosophischen Horizont, der mit Plutarch vergleichbar wäre; seine symbolischen Deutungen

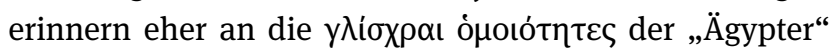
und sind oft ebenso künstlich konstruiert. Aber wenn man an die Wirkung des Büchleins denkt, läßt sich doch eine Beziehung denken. Ein Christ, der diese Fülle von Deutungen las, lernte eine Fülle von Sinnzusammenhängen kennen; die Tierwelt wurde zu einem Spiegel der christlichen Heilsgeschichte, so wie Plutarch darin die göttliche Weltordnung gespiegelt sah. Es ist vielleicht dieses Evidenzerlebnis, das hinter dem ungeahnten Erfolg des Buches im Mittelalter steht.

\subsection{Juden}

Ein zweiter Bereich, in dem Entwürfe einer religiösen Tiersymbolik entwickelt wurden, ist das hellenistische Judentum. Der Grund ist leicht zu verstehen: Die Speisegesetze des Pentateuch mit ihrer Unterscheidung der reinen und unreinen Tiere waren für die griechische Umwelt unverständlich. Die Tiersymbolik sollte helfen sie zu erklären. Unsere zwei Quellen dafür stammen aus Ägypten und es ist zu vermuten, daß die symbolischen Tierdeutungen der „Ägypter“ die Anregung dazu gaben.

Die erste Quelle ist eine Passage im Aristeasbrief (142169), welcher im 2. Jh. v. Chr. entstanden ist. Der Verfasser holt weit aus (143-145):

Allgemein gesprochen stehen alle Dinge, wenn man sie im Blick
auf die Natur versteht, in Analogie zueinander; sie werden von
einer einzigen Macht gelenkt, und in jedem Einzelfall hat es ei-
nen tiefen Sinn, daß wir von dem einen uns enthalten müssen,
das andere genießen dürfen. Beispielshalber will ich ein oder
zwei Fälle durchgehen und dir erklären. Denn du darfst nicht
in die unhaltbare Auffassung verfallen, daß Mose um der Mäu-
se oder Wiesel willen sich die Mühe gemacht hätte, diese Geset- 
ze zu erlassen; vielmehr sind diese feierlichen Vorschriften zum Zweck einer frommen Betrachtung und zur Besserung unseres Verhaltens im Sinne der Gerechtigkeit gegeben. ${ }^{29}$

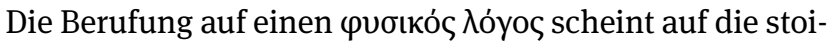
sche Lehre zu deuten, daß die ganze Natur vom göttlichen Logos durchdrungen und organisiert ist. Darauf beruht die „Ähnlichkeit“ aller Dinge (ö $\mu$ oı $\alpha$ ), das Analogieverhältnis, das symbolische Beziehungen möglich macht. (Es ist an

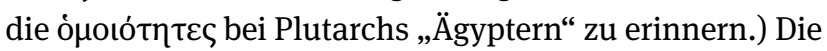
Tiersymbolik ist also mit stoischen Begriffen begründet, ähnlich wie Plutarch es mit platonischen tut.

Was die Speisegesetze betrifft, so wird ihr Sinn moralisch-erzieherisch gedeutet, sie sind ,um der Gerechtigkeit willen“ da. So sind von den Vögeln die zahmen, pflanzenfressenden rein, die Raubvögel unrein, weil sie das Prinzip von Unrecht und Gewalt verkörpern. Einhufige Tiere sind unrein, spalthufige rein: Die Spaltung symbolisiert die Unterscheidung von Gut und Böse (oder alternativ: Die Absonderung der Juden von den Heiden). Wiederkäuer sind rein, weil das Wiederkauen auf ein geduldiges Wiederholen deutet, wodurch etwas Gelerntes eingeprägt wird. Eine verblüffende Deutung bekommt das Wiesel, ein unreines Tier: Es empfängt im Ohr und gebiert aus dem Mund; das bedeutet den Spitzel, der Erlauschtes in boshafter Weise zur Anzeige bringt. Der Verfasser hat ein traditionelles Motiv mit einer offenbar in Alexandrien aktuellen politischen Spitze versehen.

Der zweite Text dieser Art ist von Philon von Alexandrien (Anfang des 1. Jh. n. Chr.): De specialibus legibus 4.100-118. Auch hier werden die Speisegesetze moralisch ausgelegt. Der Leitgedanke ist Bekämpfung der Leiden-

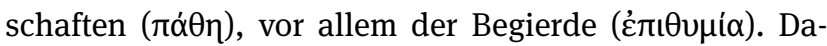
hinter steht die stoische Ethik. So sind besonders wohlschmeckende Fleischsorten verboten: Das Schwein und „schuppenlose Fische“, womit wohl die Muräne, der Luxusfisch der Römer, gemeint ist. Bezüglich der Raubtiere hat Philon einen komplizierten, fast paradoxen, Gedankengang. An sich wäre es gegenüber menschenfressenden Tieren eine gerechte Vergeltung, wenn man sie tötet und ißt (eine Erinnerung an die tierethischen Überlegungen Theophrasts). Aber das wäre eine Rachehandlung im Zorn

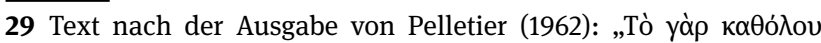

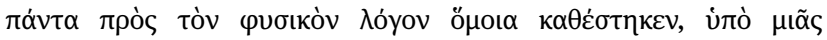

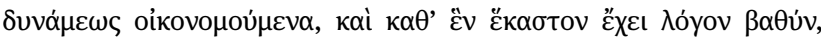

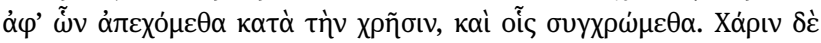

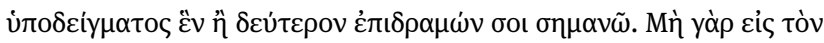

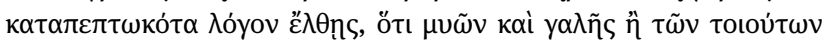

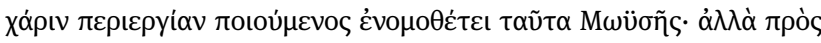

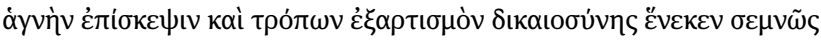

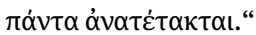

und zur Bekämpfung dieses ráӨo ist eben dies verboten! Das Verbot ist dann auf alle Raubtiere ausgedehnt. Über Spalthufer und Wiederkäuer sagt Philon Ähnliches wie der Aristeasbrief. Schließlich ein Beispiel für reine Symbolik: Wassertiere ohne Flossen sind unrein, denn sie lassen sich einfach treiben und symbolisieren damit ein hedonistisches Leben.

Die Verwandtschaft zwischen den zwei Texten zeigt, daß es im alexandrinischen Judentum eine Tradition von solchen Deutungen gegeben hat. ${ }^{30}$ Ein auffallender Zug ist ihnen gemeinsam: Viele Gedankengänge sind kühn, paradox, sie wirken gewollt geistreich, sogar spielerisch. Vielleicht ist es kein Zufall, daß manche Abschnitte des Physiologus einen ähnlichen Eindruck machen.

\subsection{Christen}

An die symbolische Auslegung der Speisegesetze auf jüdischer Seite knüpft offenbar der frühchristliche Barnabasbrief an. Sein Hauptthema ist die Absetzung des Christlichen vom Jüdischen, und in diesem Sinne werden in Kap. 10 die Speisegesetze behandelt. Die Juden halten sie fälschlich für praktische Vorschriften, aber Mose hat sie nur symbolisch gemeint. Während Aristeas und Philon die Gesetze durch die Symbolik nur erklären, will Barnabas sie ganz aufheben; die Juden hätten sie von Anfang an mißverstanden; in Wirklichkeit seien es rein moralische Mahnungen. ${ }^{31}$ Das Verbot, eine bestimmte Tierart zu essen, bedeute eine Mahnung, mit Menschen einer bestimmten Art nicht umzugehen, um ihnen nicht ähnlich zu werden.

Die in Kap. 10 behandelten Beispiele haben formale und inhaltliche Berührungen mit dem Physiologus, aber auch deutliche Abweichungen. ${ }^{32}$ Von Abhängigkeit im Sinne einer Quelle kann man nicht sprechen; eher hat man den Eindruck von Variationen über ein gemeinsames Thema. Es scheint, daß in christlichen Gemeinden ein Interesse für solche Deutungen verbreitet war und die beiden Schriften einzelne Motive daraus aufgegriffen haben.

30 Auch die Argumentation des Paulus (1 Kor 9,9-10) „Kümmert sich Gott etwa um die Ochsen?“ erinnert an diese symbolischen Deutungen.

31 Es wird oft betont, daß die Tiersymbolik der allegorischen oder typologischen Schriftdeutung nahesteht; Alpers (1984, 42-46) bevorzugt den letzteren Begriff, weil dabei der Literalsinn nicht aufgehoben werde. Bei Barnabas wird zwar nicht die Realität des Tieres, aber der Literalsinn der biblischen Gesetze aufgehoben.

32 Im Kommentar von Prostmeier (1999) sorgfältig berücksichtigt, vor allem 382-383 und 405. 
Drei Beispiele seien besprochen: die Hyäne, das Wiesel und das Motiv des „nicht schwimmenden Tieres“. Bei der Hyäne wird der Geschlechtswechsel in Barnabasbrief 10.7 und Physiologus 24 fast wörtlich gleich beschrieben. Im Barnabasbrief ist das ein Bild für den Ehebrecher

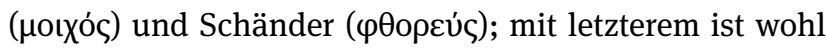
der Schänder einer Jungfrau gemeint. Der Physiologus dagegen bezieht den Geschlechtswechsel auf Bisexualität. Das ist einleuchtend, während die erstere Deutung nicht recht paßt. Es scheint, daß Barnabas die Kategorien der Sexualethik verwechselt oder jedenfalls unscharf bezeichnet.

Das Wiesel ist nach Barnabasbrief 10.8 „im Mund schwanger“; das entspricht der legendären Mundgeburt. Symbolisch ist das zu beziehen auf die, „die, wie wir hören, Gesetzlosigkeit ausüben im Mund aus Unreinheit“

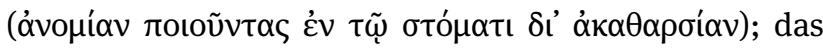
dürfte eine verdeckende Umschreibung für oralen $\mathrm{Ge}$ schlechtsverkehr einer Frau sein, in expliziter Sprache lateinisch fellatio, griechisch $\lambda \varepsilon \sigma \beta ı \alpha ́ \zeta \varepsilon ı v$. In der Gemeinde kennt man diese Art Unzucht gar nicht, deshalb der distanzierende Zusatz „wie wir hören“. Daß es sich um Frauen (Prostituierte?) handelt, geht daraus hervor, daß im folgen-

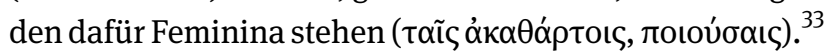
Der Verfasser stellt sich also vor, daß der Mundgeburt ein Mund-Coitus vorangegangen ist. Anders der Physiologus (Kap. 21): Das Wiesel zeugt im Mund (wie im Barnabasbrief), aber gebiert aus dem Ohr; das wird gedeutet auf einen Gläubigen, der in der Kirche das geistige Brot, den Glauben, aufgenommen hat, es aber später aus den Ohren wieder hinauswirft, also abtrünnig wird. Das wirkt sehr künstlich; Mund und Ohr kommen auch bei Plutarch vor (De Iside et Osiride 381A), aber in umgekehrter Funktion.

Eine Querverbindung zwischen dem Ibis-Kapitel des Physiologus (Kap. 40) und einigen Meerestieren des Barnabasbriefs (Kap. 10.5) entsteht durch das Motiv des NichtSchwimmens. Vom Ibis heißt es in Physiologus, „er kann

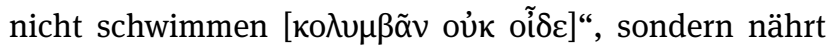
sich am Rand der Flüsse und Seen und kann nicht in die Tiefe ( $\tau \dot{\alpha} \beta \alpha \dot{\theta} \theta \eta)$ gelangen, wo die reinen Fische schwim-

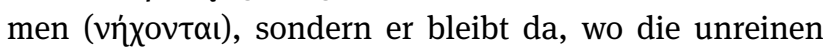

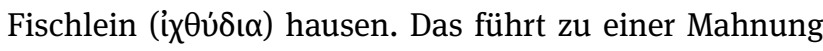
an die Christen, in die Tiefen der Gotteserkenntnis zu

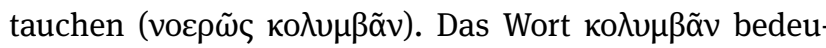
tet im engeren Sinn „tauchen“ (z. B. vom Schwammtaucher), wird aber im späteren Griechisch allgemeiner für

33 Die abweichende Deutung, es handele sich um frevlerische Reden (Prostmeier 1999, 400-401) paßt vor allem nicht zu dem Verbum

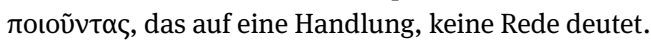

„schwimmen“ gebraucht. ${ }^{34}$ Im Barnabasbrief heißt es von drei Tieren, nämlich Muräne, Polyp und Tintenfisch, diese „Fischlein“ (ix $\theta \dot{\delta} \delta ı \alpha$ ) seien verflucht und schwömmen in

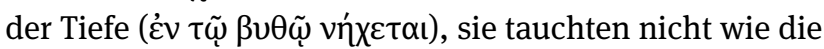
anderen, sondern wohnten auf der Erde unter der Meeres-

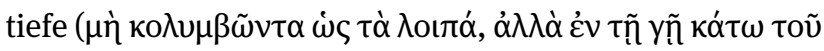

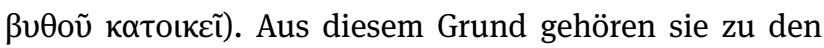
unreinen Tieren der Bibel. Hier gilt der Meeresboden als schmutzig und unrein; der Aufenthalt dort wird zwar als

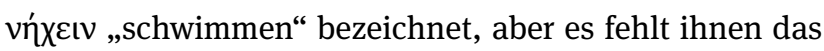

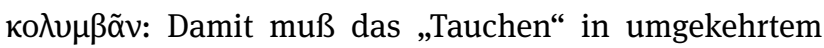
Sinn, ein Emporschwimmen von unten gemeint sein. Die zwei Abschnitte sind verbunden durch eine Verachtung der am Boden der Gewässer lebenden Tiere; einmal ist die Tiefsee, das andere Mal das Flachwasser gemeint; einmal geht es um die Tiere selbst, einmal um den Ibis, der von ihnen lebt. Auch sonst fallen Anklänge des Vokabulars auf: $\beta v \theta$ ó und $\beta \alpha \dot{\theta} \theta \eta$; das abschätzige Deminutivum ix $\theta v \dot{\delta} \iota \alpha$. Übereinstimmende Motive und Vokabeln werden also in verschiedener Weise kombiniert. Was die Wertungen betrifft, so enthält der Physiologus, ähnlich wie im Fall des Nachtraben, ein Element des Paradoxen: der Ibis, in Ägypten ein heiliger Vogel, wird abgewertet, der Nachtrabe aufgewertet.

Die Nähe des Barnabasbriefes zum Physiologus zeigt sich abgesehen von diesen Einzelfällen auch in allgemeinen Dingen:

1. Formal nähert sich die Besprechung der Tierarten dem Katalogcharakter mit jeweiligen Anfangs-Stichworten, während die zwei jüdischen Quellen eher versuchten, Sachgruppen zu bilden.

2. Es finden sich in Bezug auf Mose mehrere $\kappa \alpha \lambda \tilde{\omega} \varsigma$ Prädikationen (Barnabasbrief 10.11: , $\kappa \alpha \lambda \tilde{\omega} \varsigma$ ع $\varepsilon \tilde{\pi} \varepsilon v \beta \lambda \varepsilon^{-}$

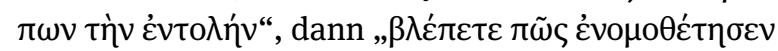

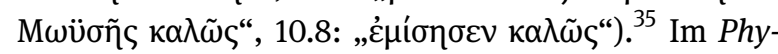
siologus wird diese Prädikation nicht nur auf die Bibel, sondern auch auf den Physiológos als Autorität bezogen, offenbar eine Erweiterung des Formelgebrauchs.

3. Die Speisegesetze werden auch im Physiologus mehrmals erwähnt, aber nur als Nebenthema.

4. Die Absetzung des Christentums vom Judentum kommt auch dort wiederholt zur Sprache, sie ist ein wesentliches theologisches Thema. Wir befinden uns anscheinend in einer Zeit, als dies noch ein aktuelles Thema in den christlichen Gemeinden war.

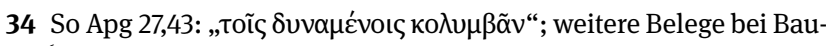
er $\left({ }^{6} 1988,899\right)$.

35 Hierzu Prostmeier (1999, 405); in Anm. 153 weist er auf Philon als Modell hin. 
Mit dem Barnabasbrief stehen wir gewissermaßen an der Schwelle zur Entstehung des Physiologus. Dessen Verfasser hat wesentliche Elemente einer Tiersymbolik vorgefunden; er hat sie aber in ein neues Konzept eingebaut. Das theologische Thema sind nicht mehr die alttestamentlichen Speisegesetze, sondern Kernfragen des Christentums: Christologie, Auferstehung und Wiedergeburt, das Leben der christlichen Gemeinde. Es werden nicht nur die Tiere der Speisegesetze besprochen, sondern allgemein biblische und auch einige nichtbiblische Tiere. Die Deutung nähert sich dem exegetischen Verfahren der Typologie an, alttestamentliche Sachverhalte als Vorausdeutung auf Christliches zu sehen. Schließlich wird „der Naturkundi-

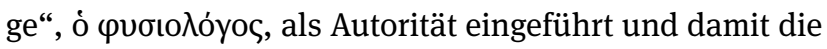
Rückbindung an die Tradition der antiken wissenschaftlichen Tierkunde ins Bewußtsein gebracht. Die Synthese dieser Elemente hat diese faszinierende Schrift hervorgebracht, die ihren Zauber erst nach anderthalb Jahrtausenden verlor.

\section{Bibliographie}

Ahrens, Karl. 1885. Zur Geschichte des sogenannten Physiologus. Ploen: S. W. Hirt's Buchdruckerei.

Alpers, Klaus. 1984. „Untersuchungen zum griechischen Physiologus und den Kyraniden. “In All Geschöpf ist Zung' und Mund: Beiträge aus dem Grenzbereich von Naturkunde und Theologie. Vestigia Bibliae: Jahrbuch des Deutschen Bibel-Archivs 6, hrsg. v. Heimo Reinitzer, 13-87. Hamburg: Friedrich Wittig.

Aubert, Hermann, und Friedrich Wimmer, Hgg. 1868. Aristoteles: Thierkunde. Bd. 1. Leipzig: Wilhelm Engelmann.

Balss, Heinrich, Hg. 1943 [2014]. Aristoteles: Biologische Schriften; griechisch und deutsch. München: Heimeran [Berlin: De Gruyter].

Bauer, Walter. ${ }^{6} 1988$. Griechisch-deutsches Wörterbuch zu den Schriften des Neuen Testaments und der frühchristlichen Literatur. Berlin: Walter de Gruyter.

Brehm, Alfred Edmund. ${ }^{2}$ 1879. Brehms Thierleben: Allgemeine Kunde des Thierreichs; Große Ausgabe. Bd. 5, Die Vögel. Leipzig: Verlag des Bibliographischen Instituts.

Ciccarese, Maria Pia. 2009. „Bibbia, bestie e Bestiari: l'interpretazione cristiana degli animali dalle origini al Medioevo.“ In Animali tra mito e simbolo. Biblioteca di testi e studi 503, hrsg. v. Sergio Botta, Alessandro Cerri, Maria Pia Ciccarese, Gilberto Mazzoleni und Adriano Santiemma, 75-124. Rom: Carocci.

Dierauer, Urs. 1977. Tier und Mensch im Denken der Antike: Studien zur Tierpsychologie, Anthropologie und Ethik. Studien zur Antiken Philosophie 6. Amsterdam: Grüner.

Dittmeyer, Leonard, Hg. 1907. Aristotelis De animalibus historia. Leipzig: Teubner.

Goldstaub, Max. (1899-1901) 1900. „Der Physiologus und seine Weiterbildung, besonders in der lateinischen und in der byzantinischen Literatur.“ Philologus: Zeitschrift für das klassische Alterthum; Supplementband 8,3:339-404.
Görgemanns, Herwig, et al. Hg. (2003) ${ }^{2} 2009$ [2014]. Plutarch. Drei religionsphilosophische Schriften. Düsseldorf: Artemis \& Winkler [Berlin: De Gruyter].

Gossen, Hans, und August Steier. 1922. „Krebs.“ PRE 11,2:16631690.

Griffiths, John G., Hg. 1970. Plutarch's De Iside et Osiride. Cardiff: University of Wales Press.

Grzimek, Bernhard. 1970. Grzimeks Tierleben. Bd. 8, Vögel II. Zürich: Kindler.

Kullmann, Wolfgang, Hg. 2007 [2009]. Aristoteles: Über die Teile der Lebewesen. Aristoteles Werke in deutscher Übersetzung 17,1, Zoologische Schriften II. Berlin: Akademie Verlag [De Gruyter].

Lambros, Spyridon P., Hg. 1885. Supplementum Aristotelicum. Bd. 1,1, Excerptorum Constantini de natura animalium libri duo: Aristophanis historiae animalium epitome. Berlin: G. Reimer.

Lauchert, Friedrich, Hg. 1889 [1974]. Geschichte des Physiologus. Strassburg: Karl J. Trübner [Genève: Slatkine].

Leroi, Armand Marie. 2014. The Lagoon. How Aristotle invented science. London: Viking.

Pelletier, André, Hg. 1962. Lettre d'Aristée à Philocrate. Sources Chrétiennes 89. Paris: Editions du Cerf.

Perry, Ben E. 1941. „Physiologus.“ PRE 20,1:1074-1129.

Perry, Ben E., Hg. 1965. Babrius and Phaedrus: Fables. Loeb Classical Library 436. Cambridge, MA: Harvard University Press.

Prostmeier, Ferdinand R., Hg. 1999. Der Barnabasbrief. Kommentar zu den Apostolischen Vätern 8. Göttingen: Vandenhoeck \& Ruprecht.

Sbordone, Francesco, Hg. 1936a. [1976, ${ }^{2}$ 1991]. Physiologus. Rom: Società Anonima Editrice ,Dante Alighieri“ [Hildesheim: Olms].

Sbordone, Francesco, 1936b. Ricerche sulle fonti e sulla composizione del Physiologus Greco, Neapel: Torella.

Schneemelcher, Wilhelm, Hg. ${ }^{6} 1997$. Neutestamentliche Apokryphen in deutscher Übersetzung. Bd. 2, Apostolisches, Apokalypsen und Verwandtes. Tübingen: Mohr Siebeck.

Sorabji, Richard. (1993) 1995. Animal Minds and Human Morals: The Origins of the Western Debate. Ithaca, NY: Cornell University Press.

Stamatiou, Aristides, und Andreas Weckwerth. 2010 „Löwe.“ RAC 23:257-286.

Thompson, D’Arcy Wentworth. (1895) ${ }^{2}$ 1936. A Glossary of Greek Birds. (Oxford: Clarendon Press) Oxford: Oxford University Press.

Wellmann, Max. 1896. „Aegyptisches.“ Hermes: Zeitschrift für classische Philologie 31,2:221-233.

Wellmann, Max. 1930. „Der Physiologos: Eine religionsgeschichtlich-naturwissenschaftliche Untersuchung." Philologus: Zeitschrift für das klassische Altertum. Supplementband 22,1:1116.

Wöhrle, Georg, Hg. 1999. Geschichte der Mathematik und der Naturwissenschaften in der Antike. Bd. 1, Biologie, Stuttgart: Franz Steiner. 